ISSN: $1130-3743$

DOI: http://dx.doi.org/10.14201/teoredu20152712532

\title{
UNA REFLEXIÓN PLURAL SOBRE EL APRENDIZAJE ÉTICO-CÍVICO MEDIADO TECNOLÓGICAMENTE
}

\author{
A pluralistic reflection on the ethical and \\ civic technologically mediated learning
}

\section{Une réflexion pluraliste sur l'apprentissage éthique et civique mediatisé technologiquement}

José Antonio IBÁÑEZ-MARTín y Juan Luis FuenTES

Universidad Internacional de La Rioja. Facultad de Educación. Gran Vía del Rey

Juan Carlos I, 41. 26002, Logroño, La Rioja.

jaimm@unir.net; juanluis.fuentes@unir.net

El último medio siglo ha estado muy influido, especialmente en Europa, por la búsqueda de la sociedad del bienestar, que tenía como objetivo no simplemente el aumento de la renta per capita sino una mejor distribución de los bienes, de modo que los servicios sociales llegaran a todos y que la calidad de vida hiciera que, por fin, pasáramos a vivir en una sociedad decente, en la que se cuidaba el medio ambiente y se respetaban todos los derechos y libertades reconocidos en la Declaración Universal de Derechos Humanos.

Si vemos en la prensa las terribles noticias de quienes mueren intentando llegar, ilegalmente, a Europa, tenemos motivos para pensar que el esfuerzo realizado estos años se ha visto coronado con el éxito: es evidente que Europa se ha convertido en un poderoso objeto de deseo. Pero, a la vez, es imposible esconder el malestar existente en la sociedad europea. La lectura de la misma prensa nos obliga a tomar conciencia de cómo son muchos los que están en desacuerdo con la política del momento, los que protestan contra la educación actual, los que consideran que sus derechos son maltratados y que debemos luchar por cambiar por completo la situación actual. De alguna manera, nos atrevemos a decir que nos encontramos ante un desencanto generalizado que, en el mejor de los casos, mueve a depositar la confianza en nuevos vientos que aparecen en la lejanía. 
Ahora bien, los nuevos vientos ¿diseñan un horizonte de un aprendizaje realmente educativo? ¿Promueven una gran política realmente preocupada por el bien común de una ciudadanía plural? ¿Animan a una reflexión filosófica profunda donde se analiza seriamente qué lleva a la plenitud humana o cuál es el horizonte que hará posible nuestro autentico desarrollo?

Naturalmente, no cabe estudiar en un solo número monográfico todas estas cuestiones. Algunos temas tocaremos sobre los vientos nuevos de la política y, sobre todo, nos dedicaremos a estudiar los vientos en el mundo de la educación actual. Pero antes de entrar en la descripción de este número, en el que junto a profesores españoles colaboran profesores de otros países, es oportuno hacer alguna consideración introductoria sobre la situación del momento en el ambiente educativo.

Resulta bastante indiscutible que el viento que actualmente predomina en el mundo de la educación es el de la empleabilidad. Hoy parece obsceno hablar de investigación básica o de la alegría del saber. No se trata de criticar cualquier iniciativa que facilite reducir los niveles de desempleo que tenemos, ni de calificar como improcedente el establecimiento de relaciones con el sistema productivo. Pero es un error importante convertir el sistema educativo en esclavo del mercado, tanto porque ello dificulta la ampliación de los futuros puestos laborales como, de modo especial, porque reduce el horizonte de la persona a su dimensión laboral, que sólo es un elemento de nuestra personalidad. Quizá, una expresión sintómatica de la fuerza de este viento la encontramos en el artículo publicado por Nasarre, antiguo portavoz de educación del Partido Popular en el Parlamento, en el que se lamenta de que el texto del Anteproyecto de Ley Orgánica para la mejora de la calidad educativa (LOMCE), presentado por su partido, que constituía el frontispicio de la justificación de la nueva ley que se proponía, comenzaba diciendo que "La educación es el motor que promueve la competitividad de la economía" (Nasarre, 2014, 14) ${ }^{1}$. Aunque este texto concreto no se aprobó, es indudable que responde a una corriente social que no puede ser considerada como una brisa transitoria, sino más bien un vendaval contra el que no es tan fácil luchar. Por eso, nos parece que, cuando surgen otros vientos, muchos acuden a ellos a ver si servirán para contrarrestar el huracán de la empleabilidad y facilitar que la nave de la educación llegue a buen puerto.

Cabría así preguntarse si, entre esos otros vientos que pudieran enfrentarse con la situación actual, se encuentra el proveniente de las tecnologías de la información y de la comunicación, el cual, basado en Internet, ha adquirido en la actualidad una fuerte presencia social. Una presencia que, sin duda alguna, tiene visos de permanecer en el tiempo, al contrario de lo que ocurría con las antiguas máquinas de enseñar.

1. El texto citado se refiere al Preámbulo del Anteproyecto de Ley Orgánica para la mejora de la calidad educativa (LOMCE), que fue modificado en su paso por las Cámaras, antes de su aprobación final. 
Pues bien, estas tecnologías ¿nos van a redimir de los males economicistas que hemos indicado? ¿Van a conseguir que seamos mejores conocedores del mundo, más capacitados para identificar los elementos básicos de lo que nos plenifica como seres humanos? ¿Van a posibilitar que mediante la comunicación seamos más dialogantes, estemos más dispuestos a cooperar, a preocuparnos de los demás? ¿Van a liberarnos de profesores con estilos anticuados que promueven no las ganas de saber sino los objetores escolares? ¿Van a lograr que seamos mejores ciudadanos, en un mundo conectado?

Evidentemente, todos deseamos que ello fuera cierto, pero no hay duda de que los nubarrones son muy grandes. Vamos, concretamente, a referirnos a siete asuntos que entendemos que tienen especial importancia en relación a esta cuestión.

1. Las redes sociales realmente, y a pesar de lo que habitualmente se piensa, no promueven el diálogo social. Así lo muestra un estudio del Pew Research Center sobre Social Media and the Spiral of Silence, hecho público en agosto del 2014, donde se señala la importancia del fenómeno descrito hace años (1974) como la espiral del silencio, que consiste en que quienes piensan que su opinión es impopular-políticamente incorrecta- tienden a callarse. Se creía que esto cambiaría con las redes sociales, por la libertad que parece otorgar la distancia, pero los estudios muestran que esa espiral aumenta, sin que se favorezca en absoluto el diálogo.

2. La indudable promoción del conocimiento que Internet consigue es irrelevante si se compara con la alta proporción de los pinchazos sobre cuestiones sexuales, así como del amplio número de este tipo de contenidos, con un clamoroso desprecio a la mujer y con especiales peligros de variado estilo para los adolescentes, que, incluso más fácilmente en la actualidad, caen en manos de los depredadores sexuales.

3. La sensación de anonimato que ha imperado en la Red ha despertado una brutalidad en las expresiones que enfangan la comunicación en toda la Red, lo que deteriora en gran medida la convivencia social, alimentando los malos instintos, contra los que todos hemos de luchar.

4. La Red no es sólo universal en el espacio sino también en el tiempo. Los errores son conocidos por todos y nunca perdonados. Ha sido muy importante la sentencia del Tribunal de la Unión Europea que obliga a Google a retirar las informaciones del pasado, si son lesivas para alguien que lo solicite, reivindicando así el derecho al olvido. Sin embargo, se trata de un proceso muy lento y complejo. De ahí que se fomenten acciones tan equivocadas como la de aquella chica que deseó tener experiencias licenciosas y que después acusó de violación a quienes atendieron sus deseos, porque temió que todo ello apareciera en las redes sociales.

5. Ha aparecido una creciente dependencia a la visibilidad como elemento estructurante de la identidad propia. El cultivo de la intimidad y de la mesura del yo están en peligro de extinción, con un claro peligro de trivializar 
la propia existencia, reduciéndola a lo que los demás ven en mí, sin que ya casi ni se pueda decir, como Don Quijote, yo sé quién soy.

6. Cada vez hay más estudios -como el publicado el año 2014 en los Proceedings of the National Academy of Sciences- que muestran que Facebook ha sido usado para analizar las consecuencias del contagio emocional, sin olvidar que el análisis de los gustos o de los lugares visitados por los internautas se crean para intensas promociones comerciales, así como los Estados interfieren los e-mails -como no pueden hacer, según las normas jurídicas de la mayoría de los países, con el correo ordinario- aduciendo razones de seguridad nacional. En suma, Internet se ha convertido en un temible instrumento de poder y de control, mucho más que de libertad. Así, Nicholas Carr (2014), autor especialmente significativo en estos ámbitos, ve en peligro la libertad ante el control ajeno de la atención, que limita y encauza las propias elecciones.

7. El atractivo de la pantalla está llevando a una confusión gravísima entre lazos realmente humanos y "amistades virtuales", aumentando la soledad verdadera. Hace años, Putnam publicó un famoso libro titulado Bowling Alone (2000) (Solo en la bolera, 2002), en el que señalaba la disminución del número de norteamericanos que se comprometían en la realización de actividades sociales, lo que expresaba con el aumento del número de personas que acudían a jugar en solitario a los bolos. Y hace un par de años, Sherry Turkle publicó un libro con el título Alone Together, en el que muestra la falsedad de quienes presentan las redes sociales como comunidad, cuando lo que consiguen es aumentar la auténtica soledad. La peor expresión de este error la encontramos en el fenómeno, que por comenzar a estudiarse en Japón ha recibido el nombre de los bikikomori, de los que se encierran en su dormitorio sin salir jamás de allí, limitando su mundo a sus presuntos "amigos virtuales". Y no deja de inquietar leer en la prensa que ya están contabilizadas varios cientos de personas en España aquejadas de la misma enfermedad.

Este monográfico, convocado desde la filosofía de la educación, pretende primeramente conocer la realidad y sus causas (felix qui potuit rerum cognoscere causas), a la vez que, obviamente, evita caer en la nostalgia y la lamentación, pues lo que busca es promover estrategias en entornos virtuales que faciliten el aprendizaje ético-cívico que exigen los nuevos tiempos, para alcanzar la plenitud a la que todos aspiramos. Para tratar estos temas, en este número incluimos diez artículos que proporcionan una perspectiva panorámica en su conjunto y profunda en su enfoque particular, con planteamientos diferentes, en algunos casos coincidentes, en otros complementarios, pero también en algún momento enfrentados, que evidencian la emergencia de un ámbito de especial interés para el conocimiento pedagógico.

Primeramente, las profesoras Concepción Naval y Elena Arbués, de la Universidad de Navarra, consideran en su trabajo la participación on line como uno de 
los posibles sustentos de las democracias en cuanto que participación ciudadana, pero que, sin embargo, posee unos requisitos que deben ser mantenidos mediante la educación cívica y la promoción de virtudes sociales. Junto a las consideraciones teóricas que incluyen la revisión crítica de algunos proyectos internacionales sobre esta específica temática, proponen actividades audiovisuales disponibles en la Red, dirigidas a los propios educadores que pueden posibilitar el logro de estos objetivos. La comprensión de virtudes de carácter social como el Respeto, la Responsabilidad, la Solidaridad o la Justicia, complementadas con metodologías activas y cooperativas, nos acercan, según las autoras, a las respuestas de preguntas sobre las razones, los fines y las vías de participación ciudadana.

También en la esfera de la participación política se encuentra el artículo de José Antonio Ibáñez-Martín (Universidad Internacional de La Rioja), quien advierte de la necesidad de renovación del sistema político democrático actual y contemporáneo de la sociedad digital, enfermo de una extendida corrupción, violencia e inmoralidad variada, que demanda el retorno a la gran política, la cual no puede comprenderse sin sus estrechos vínculos a la filosofía y la educación. Para él, el pensamiento nietzscheano posibilita delinear este concepto mediante sus críticas al obrero filosófico, a las limitaciones de la educación historicista y escéptica y a la cultura mediocre, constituyendo una base de la que manan diversas propuestas concretas para la supervivencia de la democracia mediática. Tales propuestas invitan al político a convertirse en un estadista, entendido en su sentido más pleno, del que tenemos buenos ejemplos a lo largo de la historia, que nos muestran su capacidad al proponer amplios horizontes vitales a sus ciudadanos.

A continuación, los profesores Gonzalo Jover, M. ${ }^{a}$ del Rosario González Martín (Universidad Complutense de Madrid) y Juan Luis Fuentes (Universidad Internacional de La Rioja) presentan una novedosa propuesta que vincula la participación ciudadana con Internet y los textos clásicos. Concretamente, abogan por una relectura de Antígona que permita explorar las categorías de lo público y lo privado, contextualizando la conversación entre las dos esferas en la situación política contemporánea, donde las TIC se han convertido en un facilitador de los movimientos sociales. La narrativa transmedia que plantean abre múltiples posibilidades educativas en las que los estudiantes pueden pensar críticamente sobre la realidad con motivo de la obra sofoclea, participar en un diálogo intercultural que supera las distancias entre individuos e incorpora lenguajes complementarios al verbal y convertirse en narradores de historias que incorporan reflexiones de carácter filosófico, lo que permite hablar de una verdadera experiencia de educación cívica y de construcción mediática de la ciudadanía.

Siguiendo la línea de conexión entre TIC y ciudadanía, se encuentra el estudio de Ángel García del Dujo, José Manuel Muñoz Rodríguez y María José Hernández Serrano, de la Universidad de Salamanca, donde se abordan las profundas modificaciones que la noción teórico-práctica de ciudadano ha experimentado con motivo de la tecnología. Alejándose de las limitaciones establecidas por la modernidad, y contrariamente a las pretensiones de algunos tratados internacionales, el ciudadano 
hoy es en realidad heterogéneo, dinámico, relacional e irreductible a las constricciones estatales. Además, exponen los resultados de un interesante proyecto internacional desarrollado en seis países europeos, dirigido al empoderamiento de colectivos especialmente vulnerables mediante el sentimiento de conexión e identidad compartida y la participación activa como creadores de contenidos que permiten las tecnologías, tomando como base la filosofía del periodismo ciudadano.

La mirada se dirige hacia la educación superior en el siguiente artículo escrito por Francisco Esteban, de la Universidad de Barcelona, y Juan Luis Fuentes, de la Universidad Internacional de La Rioja, cuyo texto analiza los elementos clave que las instituciones universitarias deben considerar ante su inmersión tecnológica, si aspiran a conservar su carácter esencial fundamentado en el cultivo de las virtudes. Según explican, estos elementos deben partir de una idea de autonomía heredera de un ejercicio de indagación en la búsqueda de referencias morales, vinculadas a una comunidad no neutral. El primero de ellos consiste en el reconocimiento de un sujeto de aprendizaje en sentido estricto, encarnado en la figura del estudiante universitario, que establece una relación educativa con una vOz autorizada intelectual y ética, donde los principios del conectivismo presentan importantes problemas. El segundo alude a la necesidad de identificar un contenido particular de aprendizaje elegido con el criterio de lo bueno, lo bello y lo sustancioso en sí mismo. Mientras que el tercero se refiere a las formas específicamente reclamadas por las relaciones universitarias.

Juan García-Gutiérrez, de la Universidad Nacional de Educación a Distancia, realiza en su artículo un estudio hermenéutico de la normativa y política comunitarias sobre la protección de la infancia frente a los riesgos de Internet, desde las primeras acciones y documentos en los años ochenta centrados en la televisión y la radiodifusión; pasando por diversos documentos e iniciativas donde se combina una fuerte preocupación por la seguridad con la intención de garantizar el derecho de acceso a la Red, considerándolo como un elemento clave de desarrollo social y cultural; hasta la iniciativa "Educación abierta" de 2013. Según explica, el modelo de protección a la infancia ha oscilado entre la preocupación por los contenidos y la preocupación por las interacciones, lo que implica atender no sólo a los riesgos sino también a las posibilidades de los entornos virtuales, cambiando el foco de la protección proporcionada por los adultos a la propia responsabilidad de los menores. Sin embargo, encuentra algunos obstáculos en el cambio de modelo que pueden llevar a perder de vista el interés ético-cívico de la experiencia en Internet, entre los que se encuentra un excesivo acento en las oportunidades laborales, empresariales y económicas que las iniciativas europeas priman, en ocasiones, por encima de las específicamente educativas.

La profesora de la Universidad Nacional Autónoma de México Leticia Landeros expone la experiencia de la educación ético-cívica en la modalidad de telesecundaria llevada a cabo en el país centroamericano durante los últimos años, que atiende especialmente a niños en situación de pobreza y dificultad social. El primer análisis de la evolución de esta asignatura abre paso a una descripción del 
fenómeno de la telesecundaria que atiende a más de un millón de estudiantes motivado por las grandes distancias y la falta de recursos. Después, se describe el proceso de elaboración de los materiales que en su versión final combina la propia educación en valores con una perspectiva que otorga mayor peso a la formación sociomoral basada en los derechos humanos y la educación cívica, que bebió de fuentes propias de países del entorno mexicano y también del contexto español. Recoge también algunos ejemplos de los recursos utilizados que incluyen dilemas kohlbergianos interactivos, casos centrados en problemas locales encaminados a la formación del pensamiento crítico o ejercicios para la adquisición de habilidades procedimentales de carácter fundamental en el funcionamiento de la democracia. Finalmente, plantea una serie de retos de relevancia para el desarrollo de propuestas con objetivos similares.

Desde Italia, Emanuele Balduzzi, del Instituto Universitario Salesiano de Venecia, estudia los Episodios de Aprendizaje Situados, como una particular forma de aprendizaje mediante dispositivos móviles susceptible de promover aprendizajes de carácter ético. Según plantea, esta metodología centrada en el aprendizaje permite la expresión creativa de los estudiantes, la interacción y colaboración grupal sobre la base de un objetivo común, así como ejercicios de reflexión personal y metacognición. Además, requiere una gran implicación del educador, cuya participación y preparación se incrementa aludiendo a aspectos tecnológicos, psicológicos, educativos y organizativos. Cuestiones relacionadas con el respeto, la responsabilidad, la generosidad, la preocupación por el trabajo bien hecho o el esfuerzo deben ser planteadas, según Balduzzi, en la utilización de estos dispositivos móviles, pero al mismo tiempo deben considerarse las dificultades que los entornos virtuales suponen para la interiorización de comportamientos estables encaminados al bien, como los que presuponen las virtudes en sentido aristotélico.

Los dos últimos trabajos se centran de manera particular en los adolescentes y su relación con las Tic. Primeramente, M. ${ }^{a}$ Ángeles Hernández Prados, Patricia López Vicent y Verónica Bautista Ortuño (Universidad de Murcia) realizan un estudio descriptivo dirigido a conocer la manera en que los estudiantes de los últimos cursos de la ESO perciben los valores característicos de los entornos virtuales; los agentes y herramientas que los transmiten y posibilitan; y la manera de vivenciarlos, es decir, su concreción práctica en el comportamiento en la propia Red. Mediante un cuestionario diseñado específicamente para esta investigación, se encuentra, entre otras cosas, que, según los encuestados, la creatividad/originalidad, el diálogo y la colaboración/participación son los valores más comunes en Internet, mientras que el considerado menos frecuente es la honestidad. La falta de interacción cara a cara y otros elementos condicionantes de la comunicación son identificados como los principales obstáculos, mientras que la familia vuelve a situarse de nuevo en este estudio como el principal agente de transmisión de valores.

Finalmente, la profesora M. ${ }^{a}$ Carmen Caro Samada, de la Universidad Internacional de La Rioja, pone la atención en el reto educativo y moral que Internet supone en cuanto que facilitador de diferentes estrategias que favorecen la 
mentira, especialmente en adolescentes. Hasta tal punto es habitual la circulación de información falsa en diferentes formatos creada por los internautas que ha adquirido denominaciones propias y se ha normalizado, resultando difícil para el usuario distinguir la realidad de la fantasía. Aborda también la cuestión desde la perspectiva de los perfiles sociales que nos sumerge en el análisis de la identidad digital, que es flexible y cambiante en la línea de la identidad líquida y múltiple del pensamiento moderno. La producción científica actual refleja que todo ello puede suponer problemas como la dificultad para establecer relaciones basadas en la confianza, la manipulación para el acceso a la intimidad de los otros o la fragmentación de la narrativa del yo, pero una corriente más optimista lo concibe como una oportunidad para la experimentación o un paso más del proceso de construcción identitaria. La educación del pensamiento crítico, la comprensión de todas las dimensiones de la responsabilidad en la Red, la gestión productiva de la identidad y la reputación digital son, según esta autora, los principales retos a los que los educadores nos enfrentamos.

Así pues, confiamos en que este monográfico permita profundizar en el estudio de los entornos virtuales desde una perspectiva escasamente atendida hasta el momento, principalmente centrada en elementos menos técnicos y más centrales al quehacer educativo, aquellos que inciden de manera especial en la formación de la persona en cuanto que persona, lo cual no puede realizarse sin una reflexión desde la teoría y la filosofía de la educación.

\section{REFERENCIAS BIBLIOGRÁFICAS}

CARr, N. (2014) Atrapados: cómo las máquinas se apoderan de nuestras vidas. Barcelona, Taurus.

NASARRe, E. (2014) Los fines de la educación. El malestar educativo. Nueva Revista, 49, 6-17. 